\title{
Evidence of diagnostic value of ferritin in patients with COVID-19
}

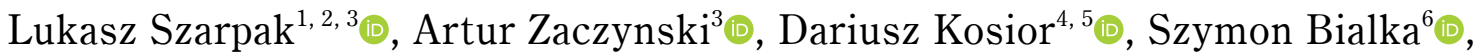 \\ Jerzy R. Ladny ${ }^{7,8}$, Natasza Gilis-Malinowska ${ }^{9}$, Jacek Smereka ${ }^{8,10}$, \\ Luiza Kanczuga-Koda ${ }^{2}$, Aleksandra Gasecka ${ }^{11}{ }^{\oplus}$, \\ Krzysztof J. Filipiak ${ }^{11}{ }^{\infty}$, Milosz J. Jaguszewski ${ }^{9}$ \\ ${ }^{1}$ Maria Sklodowska-Curie Medical Academy in Warsaw, Poland \\ ${ }^{2}$ Maria Sklodowska-Curie Bialystok Oncology Center, Bialystok, Poland \\ ${ }^{3}$ Central Clinical Hospital of the Ministry of the Interior and Administration in Warsaw, Poland \\ ${ }^{4}$ Department of Cardiology and Hypertension with Electrophysiological Lab, Central Clinical Hospital \\ of the Ministry of the Interior and Administration in Warsaw, Poland \\ ${ }^{5}$ Faculty of Medicine, Collegium Medicum, Cardinal Stefan Wyszynski University, Warsaw, Poland \\ ${ }^{6}$ Department of Anesthesiology and Intensive Care, Medical University of Silesia, Katowice, Poland \\ ${ }^{7}$ Chair of Emergency Medicine and Disaster, Medical University in Bialystok, Poland \\ ${ }^{8}$ Polish Society of Disaster Medicine, Warsaw, Poland \\ ${ }^{9}$ First Department of Cardiology, Medical University of Gdansk, Poland \\ ${ }^{10}$ Department of Emergency Medical Service, Wroclaw Medical University, Wroclaw, Poland \\ ${ }^{11}$ First Chair and Department of Cardiology, Medical University of Warsaw, Poland
}

\section{This paper was guest edited by Prof. Togay Evrin}

The current severe acute respiratory syndrome coronavirus 2 (SARS-CoV-2) pandemic forces medical personnel to search for alternative early diagnosis methods of the patient's condition [1]. An essential element of the diagnosis of a patient with COVID-19 is to understand the impact of various laboratory tests on the severity of the disease. An example of this research can be determining the level of ferritin, which is considered an indicator of the body's iron supply. As iron levels fall, the blood ferritin levels fall [2]. The concentration of $1 \mu \mathrm{g} / \mathrm{L}$ corresponds to $8 \mathrm{mg}$ of iron in the reserve pool [3]. When healthy, $20 \%$ of the body's iron is bound to ferritin. Ferritin bound iron accounts for $95 \%$ of the hepatic iron stores. According to World Health Organization, adult women's norm ranges from 15 to $150 \mu \mathrm{g} / \mathrm{L}$, for men from 15 to $200 \mu \mathrm{g} / \mathrm{L}$ [4]. Moreover, ferritin is considered an acute phase protein, so its concentration also increases inflammation and infections. This limits the possibility of using its determination to assess systemic iron resources, even in terms of the diagnosis of COVID-19 severity [5, 6]. Abbaspour et al. [7] showed that ferritin is a crucial mediator of immune dysregulation via direct immune-suppressive and pro-inflammatory effects, contributing to cytokine storm.

The present study aimed to determine the usefulness of ferritin as a predictor of a patient's severity with COVID-19 in a performer systematic review and meta-analysis. This study followed the Preferred Reporting Items for Systematic Reviews and Meta-Analysis (PRISMA) guidelines [8] and the Meta-analysis Of Observational Studies in Epidemiology (MOOSE) guidelines for reporting systematic reviews and meta-analyses of observational studies [9].

Three authors (L.S., J.S., and S.B) independently searched relevant literature. The current Pubmed, Embase, Cochrane, Web of Science, Scopus (from database inception to November 10, 2020) was explored. The whole search strategy used free words, including "ferritin" AND "COVID-19"

Address for correspondence: Lukasz Szarpak, Assoc. Prof. PhD, MBA, Maria Skłodowska-Curie Medical Acedemy in Warsaw, ul. Solidarności 12, 03-411 Warszawa, Poland, tel: +48 500186225, e-mail: lukasz.szarpak@gmail.com 


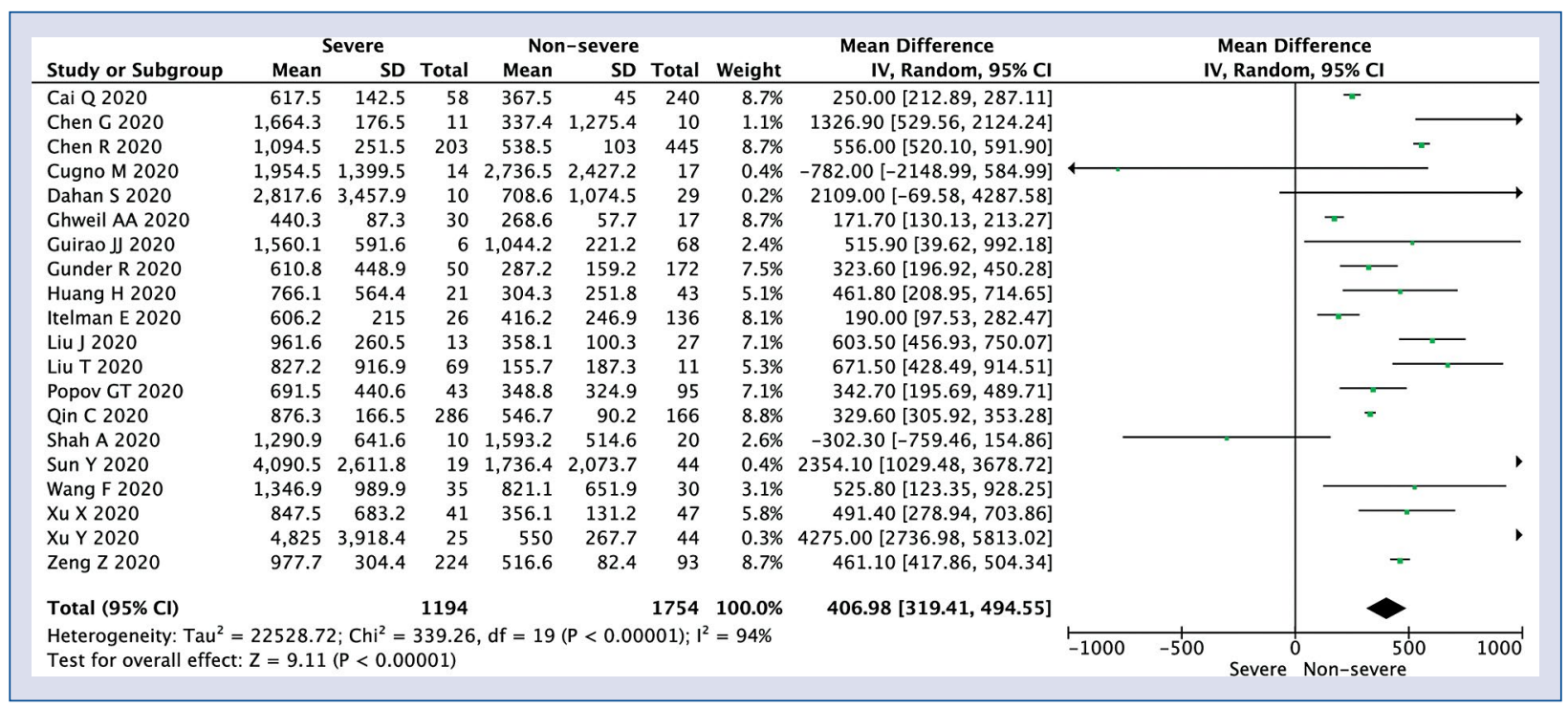

Figure 1. Forest plot of ferritin levels in severe versus non-severe groups. The center of each square represents the odds ratio for individual trials, and the corresponding horizontal line stands for a $95 \%$ confidence interval (Cl). The diamonds represent pooled results; SD — standard deviation.

OR "SARS-CoV-2". The reference lists of all eligible trials and reviews were screened for additional citations. Publications were restricted to the English language.

Twenty studies reported ferritin levels in severe and non-severe patient groups. Polled analysis showed that it significantly correlated higher ferritin levels with the more severe condition of the COVID-19 patient (MD: 406.98; 95\% CI: 319.41-494.55; $\mathrm{p}<0.001$; I': 94\%; Fig. 1). Detailed characteristics of the studies included in the meta-analysis are presented in Supplementary Digital Content. Higher ferritin levels were also associated with more frequent hospitalization in intensive care unit conditions (MD: 748.96; 95\% CI: 444.45-1053.48; $p<0.001 ; I^{2}: 89 \%$ ), and higher mortality in COVID-19 patients (MD: $594.43 ; 95 \%$ CI: 345.7-843.17; $p<0.001 ; I^{2}: 99 \%$; Supplementary Digital Content).

In conclusion, this systematic review and meta-analysis show a close correlation between ferritin levels and the state of the COVID-19 patient. Higher ferritin levels were associated with a more severe patient condition, more intensive care unit exposure, and higher mortality.

\section{Acknowledgements}

Study supported by the ERC Research NET and Polish Society of Disaster Medicine.

Conflict of interest: None declared

\section{References}

1. Dzieciatkowski T, Szarpak L, Filipiak KJ, et al. COVID-19 challenge for modern medicine. Cardiol J. 2020; 27(2): 175-183, doi: 10.5603/CJ.a2020.0055, indexed in Pubmed: 32286679.

2. Evrin T, Demirel B, Szarpak L, et al. Galectin-3: a novel blood test for the classification of patients with COPD. An observational study. Dis Emerg Med J. 2019; 4(3): 77-82, doi: 10.5603/ demj.a2019.0016.

3. Salman Z, Yılmaz T, Mehmetçik G. The relationship between ferritin levels and oxidative stress parameters in serum of $\beta$-thalassemia major patients. Arch Biochem Biophys. 2018; 659: 42-46, doi: 10.1016/j.abb.2018.09.020, indexed in Pubmed: 30287235.

4. Dopsaj V, Martinovic J, Dopsaj M, et al. Gender-specific oxidative stress parameters. Int J Sports Med. 2011; 32(1): 14-19, doi: 10.1055/s-0030-1267930, indexed in Pubmed: 21086243.

5. Henry BM, de Oliveira MH, Benoit S, et al. Hematologic, biochemical and immune biomarker abnormalities associated with severe illness and mortality in coronavirus disease 2019 (COVID-19): a meta-analysis. Clin Chem Lab Med. 2020; 58(7): 1021-1028, doi: 10.1515/cclm-2020-0369, indexed in Pubmed: 32286245.

6. Katipoğlu B, Sönmez LÖ, Vatansev H, et al. Can hematological and biochemical parameters fasten the diagnosis of COVID-19 in emergency departments? Dis Emerg Med J. 2020, doi: 10.5603/ demj.a2020.0039.

7. Abbaspour N, Hurrell R, Kelishadi R. Review on iron and its importance for human health. J Res Med Sci. 2014; 19(2): 164-174, indexed in Pubmed: 24778671.

8. Shamseer L, Moher D, Clarke M, et al. Preferred reporting items for systematic review and meta-analysis protocols (PRISMA-P) 2015: elaboration and explanation. BMJ. 2015; 350: g7647, doi: 10.1136/bmj.g7647, indexed in Pubmed: 25555855.

9. Stroup DF, Berlin JA, Morton SC, et al. Meta-analysis of observational studies in epidemiology: a proposal for reporting. Meta-analysis Of Observational Studies in Epidemiology (MOOSE) group. JAMA. 2000; 283(15): 2008-2012, doi: 10.1001/ jama.283.15.2008, indexed in Pubmed: 10789670. 